\title{
Choices in Knowledge-Base Retrieval for Consumer Health Search
}

\author{
Jimmy $^{1,3(\bowtie)}$, Guido Zuccon ${ }^{1}$, and Bevan Koopman ${ }^{2}$ \\ 1 Queensland University of Technology, Brisbane, Australia \\ jimmy@hdr.qut.edu.au, g.zuccon@qut.edu.au \\ 2 Australian E-Health Research Center, CSIRO, Brisbane, Australia \\ bevan.koopman@csiro.au \\ 3 University of Surabaya (UBAYA), Surabaya, Indonesia
}

\begin{abstract}
This paper investigates how retrieval using knowledge bases can be effectively translated to the consumer health search (CHS) domain. We posit that using knowledge bases for query reformulation may help to overcome some of the challenges in CHS. However, translating and implementing such approaches is nontrivial in CHS as it involves many design choices. We empirically evaluated the impact these different choices had on retrieval effectiveness. A state-of-the-art knowledge-base retrieval model - the Entity Query Feature Expansion model — was used to evaluate the following design choices: which knowledge base to use (specialised vs. generic), how to construct the knowledge base, how to extract entities from queries and map them to entities in the knowledge base, what part of the knowledge base to use for query expansion, and if to augment the KB search process with relevance feedback. While knowledge base retrieval has been proposed as a solution for CHS, this paper delves into the finer details of doing this effectively, highlighting both pitfalls and payoffs. It aims to provide some lessons to others in advancing the state-of-the-art in CHS.
\end{abstract}

\section{Introduction and Related Work}

A major challenge for users in consumer health search (CHS) is how to effectively represent complex and ambiguous information needs as a query $[13,14,16]$. Studies on query formulation in CHS have shown that consumers struggle to find effective query terms [14], often submitting layman and circumlocutory descriptions of symptoms instead of precise medical terms [17]. For example, people search for "skin irregularities" instead of "skin lesions" (the correct medical term for the symptom). This leads to poor retrieval effectiveness and low user satisfaction. Different approaches have been proposed to improve CHS, including query suggestion [15], learning-to-rank using syntactic, semantic or readability features $[7,12]$, and query expansion or reformulation [8-10].

Here we focus on overcoming the CHS problem by expanding/reformulating a health query with more effective terms (e.g., less ambiguous, synonyms, etc.). Manually replacing query terms with those from medical terminologies

(C) Springer International Publishing AG, part of Springer Nature 2018

G. Pasi et al. (Eds.): ECIR 2018, LNCS 10772, pp. 72-85, 2018.

https://doi.org/10.1007/978-3-319-76941-7_6 
(e.g., UMLS) has proven effective [8]. This shows that query reformulation in the CHS can be effective-but can it be done automatically?

In the general search domain, there have been a number of automated query reformulation approaches that link queries to entities in a knowledge base (KB) such as Wikipedia and Freebase and then used these related entities for query expansion. Bendersky et al. [1] approach involved linking the query to concepts in Wikipedia. Concepts from the query, denoted $\kappa_{Q}$, were weighted; the same was done for concepts in each of the documents in the corpus, denoted $\kappa_{D}$. The relevance score $\operatorname{sc}(Q, D)$ between query $Q$ and document $D$ was calculated as a relatedness measure between $\kappa_{Q}$ and $\kappa_{D}$ [1]. Later, the Entity Query Feature Expansion model [2] extended this by automatically expanding queries by linking them to Wikipedia. Instead of just using entities from Wikipedia (as Bendersky et al. [1] did), the Entity Query Feature Expansion model labelled words in the user query and in each document with a set of entity mentions $M_{Q}$ and $M_{d}[2]$. Each entity mention was related to KB entities $e \epsilon E$, with different relationship types. The queries were expanded by including entity aliases, categories, words, and types from Wikipedia articles. The expanded query was then matched against documents in the corpus using the query likelihood model with Dirichlet smoothing.

We posit that this Entity Query Feature Expansion model would have merit in CHS. It provides a means of mapping health queries to health entities in a health related (subset of a) KB, be this either a general KB (Wikipedia) or a specialised one (e.g., UMLS). The initial query can then be expanded based on related entities. In this paper, we investigated the use of both a specialised health KB, in line with previous work that expanded queries using, e.g., MeSH or UMLS $[3,9,10]$, and of a general KB like Wikipedia. Our rationale for this latter choice was the observation that consumers tend to submit queries using general terms and that these are covered by Wikipedia entities. However, Wikipedia also covers many of the medical entities found in specialised medical KBs. More importantly, there are links between the general and specialised entities in Wikipedia - links that can be exploited for query expansion. Thus, we adopted the Entity Query Feature Expansion model for our empirical evaluation, determining if such a $\mathrm{KB}$ retrieval approach is effective for CHS.

In investigating the effectiveness of the $\mathrm{KB}$ retrieval approach to CHS there are a number of important design decisions. The impact of these different decisions has not been thoroughly considered when describing the proposed approach $[1,2]$. Therefore, in this paper we also seek to empirically evaluate the impact of a number of different choices in KB retrieval for CHS: (i) KB construction; (ii) entity mention extraction; (iii) entity mapping; (iv) source of expansion; (v) use of relevance feedback. We also determine whether the use of a specialised $\mathrm{KB}$ is preferred over a general one, or vice versa.

\section{Expansion Model}

We implemented the Entity Query Feature Expansion model for retrieval on both the Wikipedia and UMLS as the KB. For the Wikipedia KB, a single 


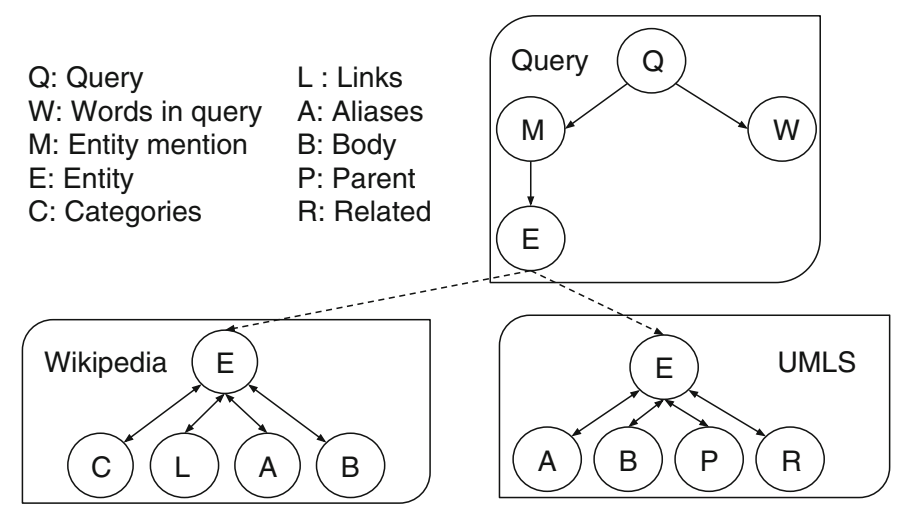

Fig. 1. Summary of expansion sources.

entity is represented by a single Wikipedia page (the page title identifies the entity). Beyond titles, Wikipedia also contains many page features useful in a retrieval scenario: entity title (E), categories $(\mathrm{C})$, links $(\mathrm{L})$, aliases $(\mathrm{A})$, and body (B). As for the UMLS KB, a single entity is represented by the most frequently used terms for a single concept unique identifier (CUI). Features of a UMLS KB entity are aliases (A), body (B), parent concepts $(\mathrm{P})$, and related concepts $(\mathrm{R})$. Figure 1 shows the features we used for mapping the queries to entities in the KB and as the source of expansion terms. We formally define the query expansion model as:

$$
\hat{\vartheta}_{q}=\sum_{M} \sum_{f} \lambda_{f} \vartheta_{f(E M, S E)}
$$

where $M$ are the entity mentions and contain uni-, bi-, and tri-gram generated from the query; $f$ is a function used to extract the expansion terms. $\lambda_{f} \epsilon(0,1)$ is a weighting factor. $\vartheta_{f(E M, S E)}$ is a function to map entity mention $M$ to the KB features EM (e.g., "Title", "Aliases", "Links", "Body", etc.) and extract expansion terms from source of expansion $S E$ (e.g., "Title", "Aliases", etc.).

\section{Choices in Knowledge Base Retrieval}

\subsection{Knowledge Base Construction}

We investigated which entities should form the basis of our KB. The CHS focus meant that health-related entities were needed. For Wikipedia KB, we considered three choices for collecting health related pages: (WC-Type) pages with Medicine infobox $^{1}$ type $^{2}$ (e.g., "abortion method", "alternative medicine", "pandemic"); (WC-TypeLinks) pages with Medicine infobox type and with links to medical terminologies such as Mesh, UMLS, SNOMED CT, ICD; (WC-UMLS) pages

\footnotetext{
${ }^{1}$ A Wikipedia Infobox is used to summarise important aspects of an entity and its relation with other articles.

${ }^{2}$ http://en.wikipedia.org/wiki/Wikipedia:List_of_infoboxes\#Health_and_fitness.
} 
with title matching an UMLS entity. The last method used QuickUMLS [11] to map Wikipedia page titles to the UMLS: if the mapping was successful, we included the Wikipedia entity (page) in the KB.

For UMLS KB, we considered two choices: (UC-All) all entities and (UCMed) entities related to four key aspects of medical decision criteria (i.e., symptoms, diagnostic test, diagnoses, and treatments) as used in [6,10]. For these choices, we included all English and non-obsolete terms.

\subsection{Entity Mentions Extraction}

Entity mention extraction is the process of identifying spans of text from the query that could map to some entity. It does not consider which exact entity (this is detailed in the next section). We considered three possible choices to extract entity mentions: (ME-All) include all uni-, bi- and tri-grams of the query (default choice); (ME-CHV) include only those uni-, bi- and tri-grams of the query that matched entities in the Consumer Health Vocabulary (CHV) $[5]^{3}$; and (MEUMLS) include only those uni-, bi- and tri-grams of the query that matched entities in the UMLS (via QuickUMLS). These three choices were used for both the Wikipedia and UMLS KBs.

\subsection{Entity Mapping}

We investigated how the entity mentions from the previous section were mapped to entities in the KB. An entity mention was mapped to an entity if an exact match was found between the mention and the entity. As shown in Fig. 1, the Wikipedia entity can be represented according to six different sources; the choices considered were: (WEM-Title) titles, (WEM-Aliases) aliases, (WEMLinks) links, (WEM-Body) the entire bodies of the Wikipedia pages, (WEM-Cat) categories, (WEM-All) all the previous sources (default choice). For UMLS KB, the choices considered were: (UEM-Title) titles, (UEM-Aliases) aliases, (UEMBody) the entire UMLS concept description, (UEM-Parent) parents, (UEMRelated) related entities, (UEM-All) all the previous sources (default choice), (UEM-QuickUmls) use QuickUMLS [11] to obtain entity mappings.

\subsection{Source of Expansion}

We investigated which sources in the $\mathrm{KB}$ were used to draw candidate terms for query expansion. We explored three choices: (SE-Title) titles associated with the entities, (SE-Aliases) aliases associated with the entities, (SE-All) both titles and aliases (default choice). While other information sources could be used (for example, those used for entity mapping), preliminary experiments showed that only these three choices produced meaningful results. These choices were used for both the Wikipedia and UMLS KBs.

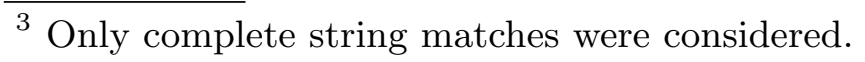




\subsection{Relevance Feedback}

We investigated the use of relevant feedback (both explicit relevance feedback (RF) and Pseudo Relevance Feedback (PRF)). We performed RF by extracting the ten most important health related words (based on tf.idf scores) from each of the top three relevant documents (relevance label greater than 0) thus resulting in a maximum of thirty expansion terms. PRF was performed by extracting the ten most important health related words from the top three ranked documents (regardless of their true relevance label). A term was considered as health related if it exactly matched a title or an alias of an entity in the target KB (either Wikipedia or UMLS).

\section{Empirical Evaluation}

To investigate the influence choices in $\mathrm{KB}$ retrieval have on query expansion for the CHS task, we empirically evaluated methods using the CLEF 2016 eHealth [18]. This collection comprises 300 query topics originating from health consumers seeking health advice online. Documents are taken from Clueweb12b13. The collection was indexed using Elasticsearch 5.1.1, with stopping and stemming. A simple baseline was implemented using BM25F with $b=0.75$ and $k 1=1.2$. BM25F allows specifying boosting factors for matches occurring in different fields of the indexed web page. We considered only the title field and the body field, with boost factors 1 and 3, respectively. These were found to be the optimal weights for BM25F for this test collection in previous work [4]. This is a strong baseline as it outperforms most runs submitted to CLEF 2016.

For constructing the Wikipedia KB, we considered candidate pages from the English subset of Wikipedia (dump 1/12/2016), limited to current revisions only and without talk or user pages. Of the 17 million entries, we filtered out pages that were redirects; this resulted in a Wikipedia corpus of 9,195,439 pages. These candidate pages were then processed according to the choices available for KB construction (Sect.3.1). Selected pages to be included in the KB were also indexed using Elasticsearch 5.1.1 with field based indexing (fields: title, links, categories, types, aliases, and body), to support the use of different fields as the source of query expansion terms (Sect. 3.4).

For constructing the UMLS KB, we indexed 3,057,234 non obsolete English terms with the following fields: title (the most frequently used term for a CUI), aliases (for all other terms used for the CUI), body (the description of a CUI), parent (title of UMLS entities with relationship type PAR), related (title of UMLS entities with relationship type RQ and RL).

Results were evaluated using nDCG@10, RBP@10 (persistence 0.5, depth 10, reporting also residuals (Res.)), in line with the CLEF 2016 collection, as users in the CHS task tend to primarily examine the first few search results. Additionally, bpref was used as a first attempt to reduce the influence of unjudged documents on evaluation (expanded queries retrieved many more unjudged documents than the baseline). In all result tables, superscripts refer to statistical significance (pairwise t-test with Bonferroni adjustment and $\alpha<0.05$ ) between the result 
Table 1. Influence of choices in KB construction; all queries (top) and high coverage queries (bottom).

\begin{tabular}{|c|c|c|c|c|c|c|}
\hline Choice & \multicolumn{2}{|c|}{ nDCG@10 bpref } & \multicolumn{2}{|c|}{ RBP@10 Res. } & \multirow[t]{2}{*}{$\overline{|e x p|}$} & \multirow[t]{2}{*}{$\langle e, g, l\rangle$} \\
\hline baseline $^{0}$ & $.2465^{1-5}$ & $.1798^{1235}$ & $.3263^{1-5}$ & .0399 & & \\
\hline WC-Type $^{1}$ & $.0950^{0245}$ & $.1485^{04}$ & $.1258^{024}$ & .7071 & 38.99 & $299,55,161$ \\
\hline WC-TypeLinks ${ }^{2}$ & $.1146^{01}$ & $.1547^{0}$ &. $\mathbf{1 5 3 2}^{01}$ & .6361 & 43.22 & $300,66,157$ \\
\hline WC-UMLS ${ }^{3}$ & $.1090^{0}$ & $.1475^{04}$ & $.1439^{0}$ & .6342 & 21.11 & $299,54,163$ \\
\hline $\mathrm{UC}^{-A l l}{ }^{4}$ & $.1256^{01}$ & $.1653^{13}$ & $.1626^{01}$ & .5976 & 29.27 & $299,63,164$ \\
\hline $\mathrm{UC} \mathrm{Med}^{5}$ &. $\mathbf{1 3 0 0} 01$ & $.1558^{0}$ & $.1552^{0}$ & .5318 & 43.83 & $270,52,151$ \\
\hline Choice & \multicolumn{2}{|c|}{ nDCG@10 bpref } & \multicolumn{2}{|c|}{ RBP@10 Res. } & $\overline{|e x p|}$ & $\langle e, g, l\rangle$ \\
\hline baseline $^{0}$ & $.4481^{5}$ & $.4700^{1345}$ & .5046 & .0010 & & \\
\hline WC-Type $^{1}$ & $.4567^{5}$ & $.4160^{05}$ & $.4342^{34}$ & .1736 & 3.54 & $13,5,6$ \\
\hline WC-TypeLinks ${ }^{2}$ & $.4816^{45}$ & $.4334^{5}$ & .4944 & .1129 & 3.54 & $13,5,6$ \\
\hline WC-UMLS ${ }^{3}$ & .4602 & $.4186^{05}$ & $.6718^{1}$ & .1814 & 17.54 & $13,9,4$ \\
\hline $\mathrm{UC}^{-A l l}{ }^{4}$ & $.4285^{25}$ & $.3791^{05}$ & $.5874^{1}$ & .0143 & 34.46 & $13,8,4$ \\
\hline${\mathrm{UC}-\mathrm{Med}^{5}}^{5}$ & $.3542^{0124}$ & $.2615^{0-4}$ & .4854 & .0466 & 46.17 & $12,6,5$ \\
\hline
\end{tabular}

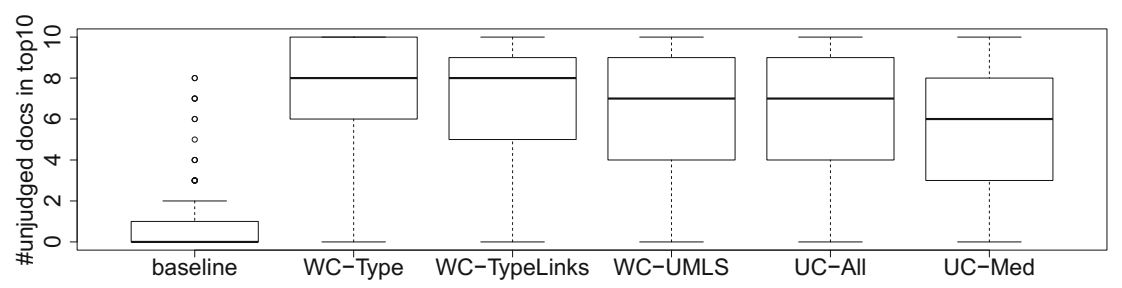

Fig. 2. Unjudged documents among the top 10 retrieved by runs in Table 1 (Top).

and the result from the choice associated with the superscript. Furthermore the average number of terms added in the expanded query $(\overline{|\exp |})$ and the number of expanded queries, queries with a gain for RBP@10 and a loss for RBP@10 were recorded as a triplet $\langle e, g, l\rangle$.

Because of space limits, for each choice, we empirically evaluated the influence the choice had on retrieval effectiveness by examining each choice sequentially. We did this across both Wikipedia and UMLS KB, and drawed conclusions about which KB best supports CHS at the end. For each choice, we fixed the best setting and use this best setting for the subsequent choice. We determined the best setting firstly based on results (i.e., nDCG@10, bpref, RBP@10) for the all queries set. If no method is clearly best for this set, then we checked results from the high coverage queries set. The complete set of results is provided in an online appendix at https://github.com/ielab/ECIR2018_KnowledgeBase_CHS.

\subsection{Knowledge Base Construction}

The effect on retrieval of choices in KB construction is reported in Table 1 (top); results are averaged over all 300 queries in the CLEF 2016 collection. 
Table 2. Influence of choices in entity mention extraction; all queries (top), high coverage queries (bottom).

\begin{tabular}{|c|c|c|c|c|c|c|}
\hline Choice & \multicolumn{2}{|c|}{ nDCG@10 bpref } & \multicolumn{2}{|c|}{ RBP@10 Res. } & $\overline{|e x p|}$ & $\langle e, g, l\rangle$ \\
\hline baseline $^{0}$ & $.2465^{1-6}$ & $.1798^{12356}$ & $.3263^{1-6}$ & .0399 & & \\
\hline WME-All $^{1}$ & $.1146^{0}$ & $.1547^{0}$ & $.1532^{0}$ & .6361 & 43.22 & $300,66,157$ \\
\hline WME-CHV ${ }^{2}$ & $.1143^{0}$ & $.1487^{04}$ & $.1573^{0}$ & .6024 & 36.06 & $285,59,155$ \\
\hline WME-UMLS $^{3}$ & $.1031^{04}$ & $.1500^{0}$ & $.1426^{0}$ & .6008 & 31.00 & $281,56,156$ \\
\hline $\mathrm{UME}^{\mathrm{A} A l}{ }^{4}$ & $.1256^{03}$ & $.1653^{25}$ & $.1626^{0}$ & .5976 & 29.27 & $299,63,164$ \\
\hline UME-CHV 5 & $.1185^{0}$ & $.1570^{04}$ & $.1539^{0}$ & .6080 & 24.85 & $288,48,168$ \\
\hline UME-UMLS 6 & $.1191^{0}$ & $.1640^{0}$ & $.1537^{0}$ & .5649 & 2.90 & $282,51,161$ \\
\hline Choice & $\mathrm{nDCGC}$ & bpref & $\mathrm{RBP} @ 10$ & Res. & $\overline{|e x p|}$ & $\langle e, g, l\rangle$ \\
\hline baseline $^{0}$ & .3218 & .3388 & .3647 & .0042 & & \\
\hline WME-All $^{1}$ & .3795 & .3286 & .4516 & .1874 & 25.32 & $22,9,8$ \\
\hline WME-CHV ${ }^{2}$ & $.3907^{3}$ & .3295 & .4714 & .1112 & .06 & $16,8,6$ \\
\hline WME-UMLS $^{3}$ & $.3606^{2}$ & .3220 & .4528 & .0652 & 22.44 & $16,8,6$ \\
\hline $\mathrm{UME}^{\mathrm{A}} \mathrm{All}^{4}$ & .3503 & .3346 & $.4162^{6}$ & .1488 & 28.62 & $21,12,7$ \\
\hline UME-CHV ${ }^{5}$ & .3466 & .3459 & .3992 & .1574 & 25.71 & $17,9,7$ \\
\hline UME-UMLS $^{6}$ & .3462 & $.3309^{4}$ & .3852 & .1256 & 23.11 & $18,9,7$ \\
\hline
\end{tabular}

The results for the Wikipedia KB showed that choice WC-TypeLinks (infobox type and links to medical terminologies) lead to the highest effectiveness across most measures. However, UC-All from the UMLS KB obtained higher effectiveness for all measures. Nevertheless, the baseline performed considerably better than the KB retrieval methods.

When further analysing the results, we found that, for a large number of queries, the $\mathrm{KB}$ retrieval methods ranked many unjudged documents amongst the top 10; while the baseline had a much lower rate of unjudged documents amongst the top 10. Figure 2 reported the distribution of unjudged documents for each of the configurations considered. This is clearly influencing the results, as demonstrated by the large values of $\mathrm{RBP}$ residuals associated with the $\mathrm{KB}$ retrieval methods in Table 1 (compared to the residual of the baseline). Interestingly, if all unjudged documents turned out to be relevant, the RBP@10 of the KB retrieval methods would prove largely superior than that of the baseline (compare the residuals).

We then considered a subset of queries for which, on average across all runs considered for a specific choice, there were a maximum of 2 unjudged documents out of the first 10 . This threshold was determined by analysing the number of unjudged documents for the baseline (the baseline does not change, irrespective of the choices), so that the threshold corresponded to 1.5 times the interquartile range above the third quartile (the upper whisker of the box-plot). Note that this produced a different subset of queries for each of the considered choices; however, the subsets had the same average "coverage" with respect to the relevance assessments. We referred to these subsets as the high coverage queries. This sub- 
Table 3. Influence of choices in entity mapping; all queries (top), high coverage queries (bottom).

\begin{tabular}{|c|c|c|c|c|c|c|}
\hline Choice & nDCG@10 & bpref & RBP@10 & Res. & $\overline{|e x p|}$ & $\langle e, g, l\rangle$ \\
\hline baseline $^{0}$ & $.2465^{1-d}$ & $.1798^{1-69 a}$ & $.3263^{1-d}$ & .0399 & & \\
\hline WEM-Title $^{1}$ & $.1547^{02569 a c}$ & $.1602^{06-a d}$ & $.1940^{025689}$ & .3699 & 25.60 & $172,32,103$ \\
\hline WEM-Aliases $^{2}$ &. $\mathbf{1 9 8 4} 4^{0134679-d}$ & $.1689^{03689 a}$ &. $\mathbf{2 6 8 1} 1^{013-79-d}$ & .2392 & 16.97 & $114,31,60$ \\
\hline WEM-Links ${ }^{3}$ & $.1506^{02569 a c}$ & $.1500^{0278 b c d}$ & $.2067^{0269 c d}$ & .3130 & 24.23 & $149,22,96$ \\
\hline WEM-Body $^{4}$ & $.1427^{025689}$ & $.1600^{0789 a d}$ & $.1826^{02589}$ & .4175 & 71.30 & $204,42,121$ \\
\hline WEM-Cat ${ }^{5}$ & $.1783^{013469-d}$ & $.1624^{06789 a d}$ & $.2320^{0124679 a c d}$ & .2673 & 25.04 & $107,22,70$ \\
\hline WEM-All $^{6}$ & $.1143^{0-5789 b d}$ & $.1487^{012578 b c d}$ & $.1573^{0-3589}$ & .6024 & 36.06 & $285,59,155$ \\
\hline UEM-Title $^{7}$ & $.1518^{0269 a c}$ & $.1774^{13-69 a}$ & $.1801^{02589}$ & .5332 & 16.82 & $287,50,160$ \\
\hline UEM-Aliases $^{8}$ & $\mathbf{. 1 7 1 7 ^ { 0 4 6 9 - d }}$ &. $\mathbf{1 8 4 7} \mathbf{7}^{1-69-c}$ & $\mathbf{. 3 6 5}^{014679-d}$ & .3633 & 9.96 & $266,75,125$ \\
\hline UEM-Body $^{9}$ & $.0734^{0-8 a-d}$ & $.1341^{0124578 b c d}$ & $.0943^{0-8 a-d}$ & .6772 & 113.14 & $296,35,180$ \\
\hline UEM-Parent $^{a}$ & $.1259^{0-35789 b}$ & $.1415^{0124578 b c d}$ & $.1702^{02589}$ & .5616 & 28.25 & $265,44,147$ \\
\hline UEM-Related $^{b}$ & $.1463^{025689 a c}$ & $.1677^{3689 a}$ & $.1915^{0289 d}$ & .5154 & 32.53 & $276,62,148$ \\
\hline UEM-All ${ }^{c}$ & $.1256^{0-35789 b}$ & $.1653^{36 a d}$ & $.1626^{023589}$ & .5976 & 29.27 & $299,63,164$ \\
\hline UEM-QuickUmls $^{d}$ & $.1355^{025689}$ & $.1792^{13-69 a c}$ & $.1563^{023589 b}$ & .5497 & 3.44 & $297,65,162$ \\
\hline Choice & nDCG@10 & bpref & RBP@10 & Res. & $\overline{\mid \overline{|e x p|}}$ & $\langle e, g, l\rangle$ \\
\hline baseline $^{0}$ & $.4018^{79}$ & $.3886^{49}$ & $.4640^{9}$ & .0017 & & \\
\hline WEM-Title $^{1}$ & $.4288^{239}$ & $.3940^{49}$ & $.4715^{9}$ & .0559 & 18.86 & $7,4,3$ \\
\hline WEM-Aliases $^{2}$ & $.3789^{179}$ & $.3850^{49}$ & $.4593^{9}$ & .0325 & 12.71 & $7,3,4$ \\
\hline WEM-Links ${ }^{3}$ & $.3655^{179 b}$ & $.3469^{9 b}$ & $.4191^{79}$ & .0619 & 33.56 & $9,3,6$ \\
\hline WEM-Body ${ }^{4}$ & $.3554^{79 b}$ & $.3289^{0125789 b}$ & $.4070^{9 b}$ & .0328 & 101.77 & $13,4,9$ \\
\hline WEM-Cat ${ }^{5}$ & $.3919^{79}$ & $.3846^{49}$ & $.4540^{79}$ & .0017 & 3.50 & $2,0,2$ \\
\hline WEM-All $^{6}$ & $.4434^{9}$ & $.3711^{9}$ & $.5412^{9}$ & .1655 & 24.00 & $15,8,6$ \\
\hline UEM-Title $^{7}$ & $.5051^{02-59}$ & $.3858^{49}$ & $.6281^{359 a}$ & .1612 & 11.10 & $20,11,8$ \\
\hline UEM-Aliases $^{8}$ & $.4250^{9}$ & $.4001^{49}$ & $.5100^{9}$ & .0438 & 15.75 & $20,12,7$ \\
\hline UEM-Body $^{9}$ & $.1752^{0-8 a-d}$ & $.2332^{0-8 a-d}$ & $.1227^{0-8 a-d}$ & .3577 & 91.81 & $21,1,17$ \\
\hline UEM-Parent $^{a}$ & $.3800^{9}$ & $.3616^{9}$ & $.4351^{79}$ & .2068 & 26.90 & $20,12,8$ \\
\hline UEM-Related $^{b}$ & $.4695^{349 c d}$ & $.4160^{349 d}$ & $.5753^{49 d}$ & .0564 & 27.10 & $21,14,6$ \\
\hline $\mathrm{UEM}^{-A} \mathrm{Al}^{c}$ & $.4114^{9 b}$ & $.3759^{9}$ & $.5075^{9}$ & .1083 & 31.43 & $21,12,7$ \\
\hline UEM-QuickUmls $^{d}$ & $.4048^{9 b}$ & $.3696^{9 b}$ & $.4615^{9 b}$ & .1818 & 27.95 & $21,10,9$ \\
\hline
\end{tabular}

set included 13 queries for choice 1 (Table 1, bottom). Results showed reduced residuals and reduced gaps between $\mathrm{KB}$ retrieval methods and the baselines; however trends in effectiveness across the considered choices for the Wikipedia KB did not change, unlike the relative effectiveness of the UMLS KB method (UC-All) that proved less effective than methods on the Wikipedia KB.

For Wikipedia, the results showed that the best setting was WC-TypeLinks. Thus, we selected WC-TypeLinks for the rest of the following analyses for Wikipedia KB; while we used UC-All for UMLS KB.

\subsection{Entity Mentions Extraction}

Table 2 (top: 300 queries and bottom: 22 high coverage queries) reports the results obtained when comparing choices for entity mention extraction. For 
Table 4. Influence of choices in source of expansion; all queries (top), high coverage queries (bottom).

\begin{tabular}{lllllll}
\hline Choice & nDCG@10 bpref & RBP@10 & Res. & $\overline{\mid e x p}$ & $\langle e, g, l\rangle$ \\
\hline baseline $^{0}$ & $.2465^{2-6}$ & $.1798^{1-4}$ & $.3263^{2356}$ & .0399 & & \\
\hline WSE-Title $^{1}$ & $\mathbf{. 2 4 2 5}^{2-6}$ & $\mathbf{. 1 8 4 3}^{023}$ & $\mathbf{. 3 2 3 0}^{2356}$ & .0829 & 1.37 & $76,26,32$ \\
WSE-Aliases $^{2}$ & $.1976^{01}$ & $.1687^{01456}$ & $.2677^{01}$ & .2376 & 16.75 & $114,30,61$ \\
WSE-All $^{3}$ & $.1984^{01}$ & $.1689^{01456}$ & $.2681^{01}$ & .2392 & 16.97 & $114,31,60$ \\
\hline USE-Title $^{4}$ & $\mathbf{. 2 1 2 6}^{0156}$ & $\mathbf{. 1 8 8 7}^{023}$ & $\mathbf{. 2 9 9 6}^{56}$ & .2119 & 2.85 & $235,73,98$ \\
USE-Aliases $^{5}$ & $.1813^{0146}$ & $.1864^{23}$ & $.2449^{014}$ & .3298 & 9.16 & $257,72,120$ \\
USE-All $^{6}$ & $.1717^{0145}$ & $.1847^{23}$ & $.2365^{014}$ & .3633 & 9.96 & $266,75,125$ \\
\hline \hline Choice & nDCG@10 & bpref & RBP@10 & Res. & $\overline{|e x p|}$ & $\langle e, g, l\rangle$ \\
\hline baseline $^{0}$ & $.2794^{1}$ & $.2189^{456}$ & $.3554^{1}$ & .0130 & & \\
\hline WSE-Title $^{1}$ & $\mathbf{. 2 8 6 0}$ & $\mathbf{. 2 2 1 1}^{45}$ & $\mathbf{. 3 7 3 7 ^ { 0 }}$ & .0149 & 1.77 & $13,8,4$ \\
WSE-Aliases $^{2}$ & .2734 & $.2191^{4}$ & .3645 & .0446 & 1.82 & $28,17,10$ \\
WSE-All $^{3}$ & .2754 & $.2191^{4}$ & .3646 & .0448 & 11.39 & $28,18,9$ \\
\hline USE-Title $^{4}$ & $\mathbf{. 2 9 2 8}$ & $\mathbf{. 2 4 0 0}^{0-3}$ & $\mathbf{. 3 8 7 0}$ & .0424 & 2.41 & $85,39,22$ \\
USE-Aliases $^{5}$ & .2633 & $.2357^{01}$ & .3578 & .0888 & 8.36 & $97,42,31$ \\
USE-All $^{6}$ & $.2619^{4}$ & $.2346^{0}$ & .3544 & .0999 & 9.11 & $99,43,32$ \\
\hline
\end{tabular}

Wikipedia, results showed that the choice of constructing entity mentions with uni-, bi- and tri-grams of the queries that matched CHV (WME-CHV) was overall the one that provided the highest retrieval effectiveness. While this is clear in the high coverage set, the difference between this strategy and using all grams (WME-All) for all queries set is less clear, probably due to the extent of many unjudged documents affecting some runs. We concluded that WME-CHV was the most effective choice and selected WME-CHV in the remaining analyses.

For UMLS, results showed that constructing entity mentions using all uni-, bi-, and tri-grams of the queries (UME-ALL) terms provided the highest retrieval effectiveness. Thus, we selected UME-ALL in the remaining analyses.

\subsection{Entity Mapping}

Table 3 (top: 300 queries and bottom: 22 queries) reports the results obtained when comparing choices for entity mapping. For both KBs, mapping entities to Aliases (WEM-Aliases and UEM-Aliases) clearly outperformed the other approaches (all queries). Results for the high coverage queries showed mixed results. Thus, we selected WEM-Aliases and UEM-Aliases for the subsequent analyses.

\subsection{Source of Expansion}

Table 4 (top: 300 queries and bottom: 119 queries) reports the results obtained when comparing sources of query expansion. Results clearly showed that selecting titles as source of expansion (WSE-Title and USE-Title) was the most effec- 
Table 5. Influence of choices in relevance feedback; all queries (top), high coverage queries (bottom).

\begin{tabular}{|c|c|c|c|c|c|c|}
\hline Choice & nDCG@10 & bpref & RBP@10 & Res. & $\overline{|e x p|}$ & $\langle e, g, l\rangle$ \\
\hline baseline $^{0}$ & $.2465^{1235-9}$ & $.1798^{478}$ & $.3263^{23689}$ & .0399 & & \\
\hline baselineRF ${ }^{1}$ & $.2055^{024569}$ & $.1777^{58}$ & $.3412^{23679}$ & .1400 & 11.70 & $150,75,74$ \\
\hline baselinePRF $^{2}$ & $.1657^{0134578}$ & $.1704^{578}$ & $.2679^{01458}$ & .2831 & 15.63 & $297,66,146$ \\
\hline GUIR-3 ${ }^{3}$ & $.1975^{02468}$ & $.1803^{8}$ & $.2636^{014578}$ & .2333 & & $292,74,134$ \\
\hline WSE-Title $^{4}$ & $.2425^{1235679}$ & $.1843^{08}$ & $.3230^{235689}$ & .0829 & 1.37 & $76,26,32$ \\
\hline WSE-TitleRF $^{5}$ & $.2133^{012469}$ & $.1833^{1268}$ &. $\mathbf{3 5 2 3} 3^{234679}$ & .1710 & 1.02 & $183,92,75$ \\
\hline WSE-TitlePRF $^{6}$ & $.1660^{0134578}$ & $.1716^{578}$ & $.2638^{01458}$ & .2928 & 16.17 & $297,71,142$ \\
\hline USE-Title $^{7}$ & $.2126^{02469}$ & $.1887^{0268}$ & $.2996^{13589}$ & .2119 & 2.85 & $235,73,98$ \\
\hline USE-TitleRF $^{8}$ & $.2245^{02369}$ & $.2006^{0-79}$ &. $\mathbf{3 6 8 7 ^ { 0 2 3 4 6 7 9 }}$ & .2290 & 9.79 & $263,94,93$ \\
\hline USE-TitlePRF $^{9}$ & $.1784^{014578}$ & $.1829^{8}$ & $.2672^{014578}$ & .2989 & 25.35 & $300,70,146$ \\
\hline Choice & nDCG@10 & bpref & RBP@10 & Res. & $\overline{|\exp |}$ & $\langle e, g, l\rangle$ \\
\hline baseline $^{0}$ & $.2718^{3678}$ & $.2309^{47}$ & $.3321^{378}$ & .0013 & & \\
\hline baselineRF ${ }^{1}$ & $.2625^{68}$ & $.2178^{78}$ &. $\mathbf{3 6 3 0}^{368}$ & .0199 & 12.00 & $38,22,16$ \\
\hline baselinePRF$^{2}$ & $.2429^{6-9}$ & $.2142^{789}$ & $.3339^{678}$ & .0662 & 15.66 & $80,25,27$ \\
\hline GUIR-3 ${ }^{3}$ & $.2363^{04789}$ & $.2207^{478}$ & $.2799^{0145789}$ & .0875 & & $79,22,29$ \\
\hline WSE-Title ${ }^{4}$ & $.2737^{368}$ & $.2378^{037}$ & $.3397^{378}$ & .0240 & 1.42 & $24,10,6$ \\
\hline WSE-TitleRF ${ }^{5}$ & $.2635^{68}$ & $.2193^{78}$ &. $\mathbf{3 6 6 9} 9^{368}$ & .0308 & 9.88 & $48,26,15$ \\
\hline WSE-TitlePRF $^{6}$ & $.2272^{01245789}$ & $.2161^{78}$ & $.3131^{125789}$ & .0932 & 15.93 & $80,24,28$ \\
\hline USE-Title $^{7}$ & $.2961^{0236}$ & $.2495^{0-6}$ & $.3981^{023468}$ & .0545 & 2.37 & $67,32,12$ \\
\hline USE-TitleRF $^{8}$ & $\mathbf{. 3 0 8 7 ^ { 0 - 6 9 }}$ & $.2445^{123}$ & $.4398^{0-79}$ & .0455 & 11.07 & $72,33,12$ \\
\hline USE-TitlePRF $^{9}$ & $.2790^{2368}$ & $.2323^{2}$ & $.3748^{368}$ & .0800 & 23.19 & $80,30,27$ \\
\hline
\end{tabular}

tive choice compared to other choices for both Wikipedia KB and UMLS KB. Therefore, we selected WSE-Title and USE-Title for the following analyses.

\subsection{Relevance Feedback}

Table 5 (top: 300 queries and bottom: 80 queries) reports the results obtained with and without relevance feedback. For Wikipedia, results showed that the addition of feedback produced mixed results. RF produced the best RBP@10 across all types of queries. In terms of nDCG@10 and bpref, the Wikipedia WSETitle choice performed better without the addition of feedback. For the UMLS, results showed that RF produced the best performance for all queries set on all measures. For the high coverage queries, the USE-Title obtained better bpref without the addition of relevance feedback. The application of relevance feedback to the baseline only improved RBP@10 when using true relevance information (RF). Nevertheless, this performed worse than the KB methods. 


\section{Further Analysis and Discussion}

In summary, we found that: (1) PRF does not improve results, independently of the KB; (2) RF instead does provide improved effectiveness, with UMLS-based best settings (USE-TitleRF) being generally better than Wikipedia-based best settings (WSE-TitleRF) for both all queries and the high coverage queries sets; (3) For the high coverage queries set (Table 5), independently of whether relevance feedback was applied, UMLS based KB best settings were more effective than Wikipedia based KB settings; for all queries set, UMLS based KB settings with RF performed better than Wikipedia based KB settings on all measures; (4) UMLS KB expanded more queries than the Wikipedia KB. This last finding is likely due to the Wikipedia KB being incomplete in that it considered only pages with health infobox and links to medical terms. Though this was the best setting, it removed many health related pages such as "headache". Further, we found that the two methods provided radically different query expansions: on average, they only had $8.9 \%$ of expansion terms in common. On top of that, we found that they retrieved different sets of documents (average overlap for the best settings without relevance feedback: $61 \%$ (55\%) of the top 1,000 (10) documents). Given these differences, we suggest future work to be directed to explore the effectiveness of combining expansions from the two KBs.

To contextualise the results obtained by KB retrieval methods, in Table 5 we also report the results of the method implemented by the GUIR-3 submission to the CLEF 2016 challenge [10]. This was the best performing, comparable ${ }^{4}$ query expansion method at CLEF 2016. The method expands queries by mapping query entities to the UMLS, and navigating the UMLS tree to gather hypernims from mapped entities as source of expansion. Post-processing is applied to the candidates to retain expansions more likely to be of benefit to retrieval. For each query, multiple expanded query variations are collected and their results aggregated using the Borda algorithm (see [10] for details). Unlike the original method, our implementation relied on BM25F rather than DFR as scoring method and QuickUMLS in place of Metamap, so as to be directly comparable with our baseline and KB retrieval methods. In Table 5 we do not report $\overline{|\exp |}$ for GUIR-3 as the method replaces some of the original terms with the expansion ones, thus making comparisons not trivial.

By observing the number of expansion terms added across the KB methods, we noted that the effective choices for KB query expansion tend to produce the lowest number of expansion terms (as well as expanding the smallest number of queries). While relevance feedback added a significant number of expansion terms (as well as expanding a large number of queries), PRF did so somewhat too aggressively, which may explain why $\mathrm{RF}$, which is more conservative both in queries that are expanded and the extent of expansion, outperformed PRF.

Finally, we analysed the results by considering the impact of query expansion for each query. Figure 3 reported the gains/losses vs. baseline obtained by the

\footnotetext{
${ }^{4}$ ECNU-2 had the highest effectiveness, but it used Google query suggestion service to gain expansions.
} 


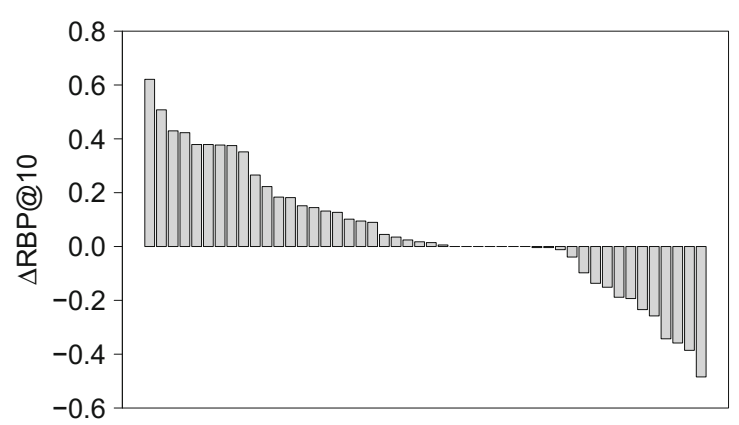

(a) Wikipedia KB

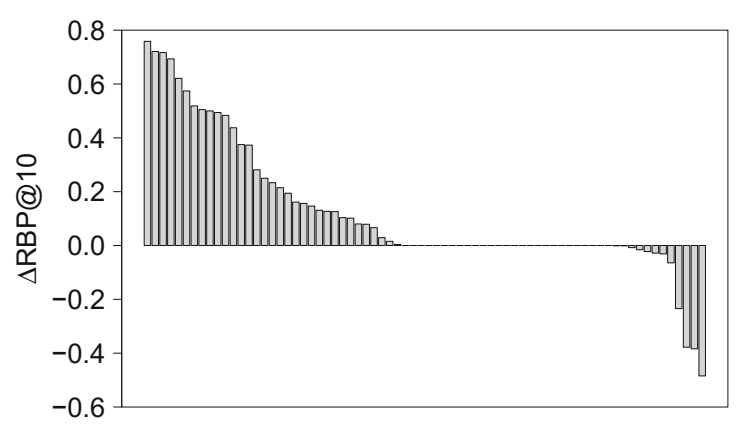

(b) UMLS KB

Fig. 3. Changes in RBP@10 between the Entity Query Feature Expansion model utilising the best settings vs. baseline. Only high coverage queries are reported.

best settings of Wikipedia KB (WSE-TitleRF) and UMLS KB (USE-TitleRF). In total, for WSE-TitleRF (USE-TitleRF), 183 (263) queries were expanded by the model (48 (72) in the high coverage set). Of these, 16 (76) showed no change in effectiveness compared to the baseline (7 (27) in the high coverage set). Of the remaining, 92 (94) showed improvements (26 (33) in the high coverage), while 75 (93) showed losses (15 (12) in the high coverage); the magnitudes of these changes are shown in the figure. These improvements (or losses) were measured using RBP@10 and thus expanded queries with low coverage are unlikely to perform as effective as expanded queries with high coverage.

\section{Conclusions}

In this paper, we explored the influence of different choices in knowledge base (KB) retrieval for consumer health search (CHS). Choices included KB construction, entity mentions extraction, entity mapping, source of expansion, and relevance feedback. We compared the effectiveness of a general KB (Wikipedia) and a medical specialised KB (UMLS) as the basis for query expansion. Our empirical evaluation showed that the best settings for the Wikipedia KB are: (1) index only Wikipedia pages that have health related infobox types or links to medical terminologies, (2) use uni-, bi-, and tri-grams of the original queries that matched CHV terms as entity mentions, (3) map entity mentions to Wikipedia entities based on the Aliases feature, (4) source expansion terms from the mapped Wikipedia page Title, and (5) add relevance feedback terms. As for the UMLS KB, the best settings are: (1) index all UMLS concepts, (2) use all uni-, bi-, and tri-grams of the original queries as entity mentions, (3) map entity mentions to UMLS entities based on the Aliases feature, (4) source expansion terms from the mapped UMLS Title feature, and (5) add relevance feedback terms.

Results after tuning the 5 choices showed that, overall, UMLS based KB settings were more effective than Wikipedia based ones. For all queries set, the best UMLS KB settings (USE-TitleRF) performed better than the baseline in terms of bpref $(+11.56 \%)$ and RBP@10 $(+12.99 \%)$. For queries with high coverage of 
judged documents, USE-TitleRF was more effective for a majority of queries and outperformed the baseline on all measures: nDCG@10 $(+12.58 \%)$, bpref $(+5.89 \%)$, and RBP@10 $(+32.43 \%)$. These results confirm that a knowledge-base retrieval approach does translate well into this often challenging CHS domain.

The major limitation of our experiments was the number of unjudged documents retrieved using the expanded queries on the CLEF 2016 collection. We mitigated this by considering bpref, RBP and RBP residuals; yet, we found challenging to fairly evaluate the methods. Nevertheless, this work provides the first thorough investigation of choices in $\mathrm{KB}$ retrieval for $\mathrm{CHS}$, highlighting both pitfalls and payoffs.

Acknowledgements. Jimmy is sponsored by the Indonesia Endowment Fund for Education (Lembaga Pengelola Dana Pendidikan/LPDP). Guido Zuccon is the recipient of an Australian Research Council DECRA Research Fellowship (DE180101579).

\section{References}

1. Bendersky, M., Metzler, D., Croft, W.: Effective query formulation with multiple information sources. In: WSDM 2012, pp. 443-452 (2012)

2. Dalton, J., Dietz, L., Allan, J.: Entity query feature expansion using knowledge base links. In: SIGIR 2014, pp. 365-374 (2014)

3. Díaz-Galiano, M., Martín-Valdivia, M., Ureña-López, L.: Query expansion with a medical ontology to improve a multimodal information retrieval system. JCBM 39(4), 396-403 (2009)

4. Jimmy, Zuccon, G., Koopman, B.: Boosting titles does not generally improve retrieval effectiveness. In: ADCS 2016, pp. 25-32 (2016)

5. Keselman, A., Tse, T., Crowell, J., Browne, A., Ngo, L., Zeng, Q.: Relating consumer knowledge of health terms and health concepts. In: AMIA 2006 (2006)

6. Limsopatham, N., Macdonald, C., Ounis, I.: Inferring conceptual relationships to improve medical records search. In: OAIR 2013, pp. 1-8 (2013)

7. Palotti, J., Goeuriot, L., Zuccon, G., Hanbury, A.: Ranking health web pages with relevance and understandability. In: SIGIR 2016, pp. 965-968 (2016)

8. Plovnick, R., Zeng, Q.: Reformulation of consumer health queries with professional terminology: a pilot study. JMIR, 6(3) (2004)

9. Silva, R., Lopes, C.: The effectiveness of query expansion when searching for health related content: Infolab at CLEF eHealth 2016. In: CLEF 2016 (2016)

10. Soldaini, L., Edman, W., Goharian, N.: Team GU-IRLAB at CLEF eHealth 2016: Task 3. In: CLEF (Working Notes), pp. 143-146 (2016)

11. Soldaini, L., Goharian, N.: QuickUMLS: a fast, unsupervised approach for medical concept extraction. In: SIGIR MedIR 2016, Pisa, Italy (2016)

12. Soldaini, L., Goharian, N.: Learning to rank for consumer health search: a semantic approach. In: Jose, J.M., Hauff, C., Altıngovde, I.S., Song, D., Albakour, D., Watt, S., Tait, J. (eds.) ECIR 2017. LNCS, vol. 10193, pp. 640-646. Springer, Cham (2017). https://doi.org/10.1007/978-3-319-56608-5_60

13. Toms, E., Latter, C.: How consumers search for health information. HIJ 13(3), 223-235 (2007)

14. Zeng, Q., Kogan, S., Ash, N., Greenes, R., Boxwala, A.: Characteristics of consumer terminology for health information retrieval. JMIM 41(4), 289-298 (2002) 
15. Zeng, Q.T., Crowell, J., Plovnick, R.M., Kim, E., Ngo, L., Dibble, E.: Assisting consumer health information retrieval with query recommendations. JAMIA 13(1), 80-90 (2006)

16. Zhang, Y.: Searching for specific health-related information in MedlinePlus: behavioral patterns and user experience. JAIST 65(1), 53-68 (2014)

17. Zuccon, G., Koopman, B., Palotti, J.: Diagnose this if you can: on the effectiveness of search engines in finding medical self-diagnosis information. In: Hanbury, A., Kazai, G., Rauber, A., Fuhr, N. (eds.) ECIR 2015. LNCS, vol. 9022, pp. 562-567. Springer, Cham (2015). https://doi.org/10.1007/978-3-319-16354-3_62

18. Zuccon, G., Palotti, J., Goeuriot, L., Kelly, L., Lupu, M., Pecina, P., Mueller, H., Budaher, J., Deacon, A.: The IR task at the CLEF eHealth evaluation lab 2016: user-centred health information retrieval. In: CLEF 2016 (2016) 